\title{
O CONTEXTO PROFISSIONAL DO INDEXADOR NO ENSINO DE INDEXAÇÃO
}

Mariângela Spotti Lopes Fujita Professora Adjunta do Departamento de Ciência da Informação da Faculdade de Filosofia e Ciências da UNESP fujita@marilia.unesp.br

\section{Resumo}

Propõe-se a investigação de metodologias de ensino com abordagem sociocognitiva para apresentação de estratégias de ensino que simulem a vivência de contextos profissionais propícios à elaboração das tarefas de análise de assunto para indexação de documentos. $\mathrm{Na}$ perspectiva do contato de indexadores aprendizes com um contexto profissional real, foram aplicadas as propostas do Portfólio de Fourie (2002) e o princípio da contextualização social de Ciência da Informação de Hjørland (2002) junto à disciplina "Indexação" do Curso de Biblioteconomia da UNESP de Marília. Os resultados obtidos desta pesquisa sobre investigação de estratégias de ensino em leitura documentária demonstram que os conhecimentos teóricos e metodológicos, em perspectiva sociocognitiva, proporcionam a visão de um contexto profissional no qual o indexador aprendiz tem consciência de suas estratégias de leitura documentária e consegue desenvolver seu conhecimento prévio profissional, de modo a realizar a tarefa de indexação compatível com a demanda informacional de usuários.

Palavras chaves: Indexação. Ensino de indexação. Abordagem sociocognitiva. Contexto profissional. 


\section{INTRODUÇÃO}

O contexto profissional do indexador é baseado em seu conhecimento quanto às demandas reais de informação da comunidade de usuários de sistemas de recuperação da informação. $\mathrm{O}$ contexto dos sistemas de recuperação da informação é uma situação, inerente à atuação profissional do indexador, que não é possível, de modo completo, ser apresentada em sala de aula ou ser vivenciada pelo aluno.

O ensino de indexação, em sala de aula, não tem condições de reproduzir o contexto de um sistema de recuperação da informação para que os alunos o vivenciem e possam transportar, para a prática de indexação de documentos, o conhecimento sobre as necessidades de busca e recuperação, sobretudo com relação ao uso de linguagens documentárias especializadas compatíveis com a linguagem de conteúdos documentários e das estratégias de busca.

Com relação à vinculação do contexto profissional em abordagem sócio-cognitiva, Fujita (2007a) constatou, em pesquisa realizada, que o indexador aprendiz necessita antever o contexto profissional real e desenvolver conhecimentos e estratégias profissionais adequadas à análise do assunto. Esse resultado foi obtido através da aplicação do protocolo verbal individual, feita em sala de aula e com alunos da disciplina "Leitura Documentária" do segundo ano do Curso de Graduação em Biblioteconomia, para observar a tarefa de leitura de um artigo científico de Ciências Biológicas sem a explanação dos procedimentos de indexação.

O objetivo da tarefa era realizar a análise de assunto para identificação de termos significativos para a indexação do artigo. A análise das dificuldades dos alunos, a partir dos dados da transcrição dos protocolos verbais, revelou a não familiaridade com o assunto do texto e termos específicos, ausência de conhecimentos teórico-práticos do processo de indexação, falta do objetivo de indexação, desconhecimento do contexto de busca de um sistema de informação com demandas reais de informação pela comunidade de usuários e falta de uniformidade de procedimentos de análise de assunto para identificação de termos representativos.

Com relação ao desconhecimento do contexto de busca, é importante considerar que o contexto dos sistemas de recuperação da informação é baseado na demanda da comunidade de usuários, uma situação inerente à atuação profissional do indexador que não é possível, de modo completo, ser apresentada ao aluno ou vivenciada por ele. Tal situação foi considerada 
previsível pela pesquisa, uma vez que a sala de aula não possuirá condições de reproduzir o contexto de um sistema de recuperação que possa ser vivenciado de modo real.

O problema que se coloca é como proporcionar ao ensino de indexação as condições metodológicas que prevejam a demanda de informação dos usuários considerando o amplo contexto de recuperação da informação em que estão inseridos todos os processos de organização da informação, incluídos aí o tratamento de conteúdo por meio da análise de assunto. Entretanto, a proposição da pesquisa é justamente a investigação de metodologias de ensino com abordagem sociocognitiva que possam apresentar estratégias de ensino que simulem a vivência de contextos profissionais propícios à elaboração das tarefas de análise de assunto para indexação de documentos.

\section{METOdologias de ENSINO SOCIOCOGNITIVAS PARA A FORMAÇÃO DO INDEXADOR}

A formação e capacitação de indexadores e resumidores deve ser questionada em estudos relacionados ao ensino com abordagem de "receita", no qual trabalhos são realizados de acordo com regras e diretrizes de livros, manuais e normas, e que, em muitas situações, o professor é um mero repetidor de teorias e práticas nas quais nunca se aprofundou cientificamente. Considerando-se que a indexação e a elaboração de resumos são processos que dependem da leitura do indexador e do resumidor, é preciso pensar antes no ensino da leitura documentária como processo cognitivo em abordagem sociocognitiva.

Como ensinamos o processo cognitivo da leitura documentária? Seremos capazes de introduzi-los em uma abordagem sociocognitiva que proponha a interação e a mediação?

Com a abordagem sociocognitiva, é possível, segundo Hjørland (2002a, p.) mudar “[...] o foco da Ciência da Informação de indivíduos (ou computadores) para o mundo social, cultural e científico" considerando-se que "[...] as ferramentas, conceitos, significado, estruturas de informação, necessidade de informação e critérios de relevância são moldados em comunidades de discurso".

Em amplo trabalho de pesquisa realizado com bibliotecas universitárias, Fujita, Rubi e Boccato (2009), o foco da investigação foi o contexto de tratamento da informação documentária do catalogador em bibliotecas universitárias. A abordagem sociocognitiva, 
fundamentada na perspectiva de Hjørland (2002b), do contexto de tratamento da informação documentária considerou:

[...] objetivos da indexação, política de indexação, regras e procedimentos do manual de indexação, a linguagem documentária para representação e mediação da linguagem do usuário e os interesses de busca do usuário, o que significa considerar o sistema de informação e seus serviços de organização e recuperação de informação, bem como o indexador com seu conhecimento prévio profissional e objetivo em situação de interação durante o processamento textual para os objetivos de indexação (FUJITA, RUBI, BOCCATO, 2009, p. 6).

A abordagem sociocognitiva, portanto, tem como foco o contexto em que o sujeito realiza uma determinada atividade em perspectiva histórica e cultural, bem como sua cognição em relação ao seu contexto de produção.

No ensino da leitura, existem implicações pedagógicas que se referem ao processo cognitivo de aprendizagem em sala de aula. Segundo Araújo (1997), as implicações pedagógicas do processo de compreensão de leitura na perspectiva interacionista entre linguagem e pensamento inferida por Goodman (1977 apud ARAUJO, 1997, p.5-6) são profundas e cobrem um largo espectro da atividade do professor e dos alunos, quais sejam:

a) No ensino da leitura, o professor deveria dar uma ênfase primordial às estratégias de compreensão, priorizando aquelas que envolvem níveis mais elevados de raciocínio;

b) Uma vez que as diferentes estratégias processuais interagem umas com as outras, cada leitor pode tirar vantagens dos seus pontos fortes, de modo a ultrapassar as suas dificuldades;

c) Não existe apenas uma via para o ensino da leitura. O professor deverá conscientizar-se de que a abordagem da leitura terá de ser eclética;

d) A seleção de materiais de leitura individualizados é extremamente útil e altamente recomendada, porque permitirá a cada aluno avançar de acordo com o seu próprio ritmo de aprendizagem.

e) Uma ênfase inicial na aceleração do ritmo de leitura dos alunos é geralmente necessária para modificar as estratégias da linearidade geradoras de dificuldades em termos de compreensão das mensagens.

f) Os temas dos materiais de leitura deverão ter as seguintes características: ser de grande interesse; estarem relacionados com o "background" do leitor; e estarem graduados em termos de dificuldade.

Em seus comentários finais sobre as concepções de análise de assunto, mais especificamente sobre a concepção orientada para a demanda, Albrechtsen (1993, p.223) levanta questões sobre a capacitação dos indexadores:

Como os indexadores podem distinguir assuntos de alta ou baixa prioridade em um documento e assegurar sua possível visibilidade em sistemas de recuperação da informação para o futuro? Quanta responsabilidade deveria ser imposta para que nós possamos julgar e mediar as qualidades de um documento para usuários potenciais?

Em sua resposta, Albrechtsen (1993) sugere uma revisão das práticas em indexação, porque geralmente são limitadas pela sua própria modéstia e livres de valores éticos na disseminação 
do conhecimento. Por isso, considera que a orientação para a demanda envolve um alto grau de subjetividade e responsabilidade ao escolher entre as qualidades do documento, face à necessidade de prudência ética na objetividade em mediar seus serviços com seus grupos alvos.

Garcia Marco (1995, p. 220), ao referir-se ao ensino universitário na área de Biblioteconomia, revela que muitos teóricos na Espanha decidiram encastelar-se em uma perspectiva unicamente profissional - em defesa da profissão, tal como se desenvolve nos postos de trabalho existentes, sujeita à evolução imposta pelos avanços realizados no estrangeiro: normalização, automação e etc. - dedicando seus melhores esforços a inventariar e descrever a prática profissional e renunciando a uma fundamentação teórica e científica das disciplinas de Arquivística, Bibliografia e Documentação.

Devemos considerar que o ensino de indexação, particularmente os processos que se realizam pela leitura documentária, precisa ser investigado por método científico de caráter aplicado, pois propiciará a formação de indexadores com o objetivo de aperfeiçoar a circulação dos conhecimentos em nossas sociedades e cujo apoio teórico se encontra distribuído nas ciências que vão desde a Lingüística, Psicologia Cognitiva e Ciência Cognitiva.

Concordamos, dessa forma, com Garcia Marco (1995, p.220), quando expõe que o ensino na área de Organização do Conhecimento deve propor-se três grandes objetivos:

a) Fundamentar o caráter científico da disciplina e formar nos alunos o uso criador e crítico do método científico; b) Proporcionar conhecimento e prática suficiente de suas principais metodologias e técnicas; c) Capacitar o aluno para o vocabulário e para modelos básicos das ciências interdisciplinares conectadas com ele.

Portanto, é necessário um ensino interdisciplinar que possa usar fontes de modelos científicos e recursos que precisam ser emprestados para o cumprimento de nossa missão de mediadores no contexto de transmissão do conhecimento socialmente acumulado e na assistência da produção de novos conhecimentos (GARCIA MARCO, 1995, p.222).

Enfatizamos a necessidade de investigação interdisciplinar para o ensino de indexação para indicar aos docentes responsáveis pelas disciplinas da área de tratamento temático da informação, cursos de Biblioteconomia, Grupos de Estudos Curriculares e Gerentes de sistemas de informação com serviços de análise, que o indexador é um leitor documentário e, portanto, precisa:

- Ter conhecimento profissional que inclua a política de indexação, linguagens documentárias, tipologias e estruturas textuais, procedimentos de análise de assunto, indexação e seus objetivos; 
- Ter conduta profissional ética, ao realizar compreensão de leitura durante a análise, para ajudá-lo no alcance de seu objetivo de indexação: o de representar fielmente o conteúdo documentário com garantia de recuperação.

Para elaborar estratégias de ensino que proponham a vinculação do contexto profissional em abordagem sociocognitiva, é preciso pensar sob duas perspectivas: como tornar possível o contato de indexadores principiantes com um contexto profissional real e, também, como desenvolver a interação cognitiva.

No que se refere à perspectiva do contato de indexadores principiantes com um contexto profissional real, Fujita (2007b) recomenda ao professor adotar as propostas do portfólio de Fourie (2003) e o princípio da contextualização social da ciência da informação de Hjørland e Albrechtsen (1995), desenvolvendo em sala de aula sob as seguintes condições:

- Explicitar o processo de indexação em perspectiva teórica e metodológica do tratamento temático de conteúdo e explorar o contexto e função da indexação em sistemas de informação;

- A aplicação do Modelo de Leitura Documentária deve ser antecedida por experiências extra-aulas de convívio com sistemas de recuperação da informação para contato com demandas reais de usuários; dar conhecimento do contexto de busca de um sistema de informação com demandas reais de informação pela comunidade de usuários; é importante considerar que o contexto dos sistemas de recuperação da informação baseados na demanda da comunidade de usuários é uma situação inerente à atuação profissional do indexador.

- O contexto e a função da indexação terá abordagem sociocognitiva, na proposta do Portfolio de Fourie (2003), propiciando ao aluno contato com o contexto real de unidades de informação mediante elaboração do trabalho de política de indexação em biblioteca ou outro sistema de tratamento e recuperação da informação para distintas áreas de assunto, bem como contato com a política de indexação vigente em manuais de indexação de sistemas de informação que produzem base de dados para realizar exercícios de indexação de artigos de periódicos em diferentes áreas de assunto;

- Com base na perspectiva de Hjørland (2002), entendemos que os alunos poderão realizar coleta de dados com usuários para obter a percepção da demanda da necessidade de informação, mediante contato com o conhecimento individual ou de grupo;

- Antes de iniciar a tarefa de leitura documentária para indexação, poderá ser realizada uma análise da tarefa, na perspectiva da abordagem sociocognitiva com textos selecionados, na qual o aluno obteve contato, em trabalhos dos itens anteriores, com o contexto da unidade de informação e da demanda do usuário de distintas áreas de assunto;

- Durante essa tarefa os alunos também poderão ter contato com a linguagem documentária específica da área do texto e utilizar dicionários especializados para completar seu conhecimento sobre a área e realizar a atividade de indexação cientes do contexto e da demanda de informação;

Os estudos de Giasson, Fourie, Hjørland e Albrechtsen contêm perspectiva sociocognitiva para propor indicações pedagógicas de estratégias de ensino que consideram o contexto como facilitador da compreensão de leitura: 
a) Modelo de ensino explícito (Giasson, 1993): apresenta as seguintes etapas: definir a estratégia e precisar sua utilidade; tornar o processo transparente; interagir com os alunos e orientá-los para o domínio da estratégia; favorecer a autonomia na utilização da estratégia e assegurar a aplicação da estratégia. O ensino explícito pode oferecer subsídios à compreensão de leitura do indexador aprendiz, enquanto realiza a indexação, com o objetivo de determinar o assunto do texto e dali identificar e selecionar conceitos.

b) Portfolio (Fourie, 2002): conjunto dos trabalhos de pesquisa do aluno, que demonstraria seus esforços e desenvolvimento na seleção de conteúdos e seus critérios, evidenciando sua autonomia e motivação para tornar-se independente no processo de aprendizagem.

c) Hjørland e Albrechtsen (1995), em “análise do domínio", consideram, como princípio, a contextualização social da ciência da informação. De acordo com o pesquisador, precisamos observar o conhecimento individual em perspectiva histórica, cultural e social. Assim, o ensino de procedimentos de análise de assunto, por meio da leitura documentária, precisa oferecer ao indexador aprendiz oportunidades de observação de um contexto sociocultural amplo, no qual tenha consciência de suas estratégias de leitura e desenvolvimento de seu conhecimento prévio profissional.

Dessa forma, é oportuno oferecer ao ensino do indexador aprendiz o acréscimo de estratégias de ensino de abordagem sociocognitiva. Ao mesmo tempo, é necessário que o ensino de indexação seja incrementado com a possibilidade de auto-observação do processo de aprendizagem pelo indexador aprendiz e participação do professor como parâmetro de experiência em indexação por meio de Protocolo Verbal.

\section{METODOLOGIA}

$\mathrm{Na}$ perspectiva do contato de indexadores aprendizes com um contexto profissional real, foram aplicadas as propostas do Portfólio de Fourie (2002) e o princípio de contextualização social da Ciência da Informação de Hjørland (2002) e Hjørland e Albrechtsen (1995), junto à disciplina "Indexação" do Curso de Biblioteconomia da UNESP de Marília.

Para isso, foi solicitada aos alunos a tarefa de levantamento do diagnóstico da política de indexação na biblioteca universitária da Universidade Estadual Paulista "Júlio de Mesquita Filho" (UNESP) no Campus de Marília, para distintas áreas de assunto, bem como análise da 
política de indexação vigente em manuais de indexação da Biblioteca Virtual de Saúde (BVS) da BIREME, como sistema de informação que produz base de dados para que, em continuidade, realizassem exercícios de indexação de artigos de periódicos em diferentes áreas de assunto. A biblioteca universitária do Campus oferece a facilidade de contato com sua estrutura e organização de domínios especializados e também é interessante pelo fato de ser o ambiente mais característico de atuação profissional do bibliotecário. Por outro lado, a BVS pode ser considerada uma referência profissional de serviço de informação especializado em ciências de saúde, cuja política de indexação e recuperação está totalmente disponível no sítio eletrônico disponível em página da web.

O portfólio de Fourie (2002) foi utilizado como metodologia para a elaboração do diagnóstico da política de indexação da Biblioteca da UNESP e da BVS da BIREME, a partir de um conjunto de trabalhos de pesquisa do aluno quanto aos contextos profissionais analisados, para colocar os alunos em contato com um contexto real de sistema de recuperação da informação.

A elaboração do portfólio teve como objetivo propiciar aos alunos a percepção de demanda da necessidade de informação, mediante contato com o conhecimento individual e do grupo em ambiente acadêmico na perspectiva de Hjørland (2002), na medida em que foram orientados a coletar dados com pesquisadores e usuários da biblioteca da UNESP e da BVS, para formar um conhecimento prévio sobre termos específicos de cada domínio, necessidades e dificuldades, tal como em um contato real com o contexto profissional de indexação.

Nesse sentido, foram realizadas duas dinâmicas: elaboração do portfólio em grupo, fora de sala de aula e indexação, dentro de sala de aula, com pares de alunos. Antes das duas dinâmicas, o professor realizou orientação do conteúdo específico de indexação com apresentação do processo e do objetivo de indexação, evidenciando aos alunos a complexidade da leitura para análise de assunto mediante observação de suas dificuldades, procedimentos e estratégias e para demonstrar a necessidade do ensino de procedimentos específicos.

1. Dinâmica em grupo fora de sala de aula - Elaboração de um portfólio que contenha informações, baseadas no Portfólio de Fourie (2002) e de Hjørland (2002), sobre:

a) a política de indexação e recuperação da Biblioteca da UNESP ou da BVS, em domínio da escolha do grupo;

b) a percepção da demanda da necessidade de informação com aplicação de entrevistas com pesquisadores de grupos de pesquisa do Campus de Marília, para contato com o conhecimento individual e do grupo em perspectiva acadêmica. 
Para obter informações sobre a política de indexação e recuperação na Biblioteca da UNESP, foi dada orientação de coleta de documentos normativos na biblioteca e informações no próprio site da biblioteca, contato com a linguagem documentária, além de entrevistas com o dirigente e o catalogador. No caso da BVS, os grupos foram orientados a fazer um levantamento e análise de documentos normativos, contato com a linguagem documentária e análise de informações complementares no sítio eletrônico disponível em página da web.

Para se obter a percepção da demanda da necessidade de informação, cada grupo foi orientado a entrevistar dois pesquisadores integrantes de um mesmo grupo de pesquisa, para estabelecer contato com o tema especializado das pesquisas do grupo de pesquisa. Antes da entrevista, o grupo de alunos teve contato com a produção científica do grupo de pesquisa através de consulta ao Diretório de Grupos de Pesquisa do CNPq e ao LATTES de cada pesquisador. Durante a entrevista, foi orientado solicitar aos pesquisadores a escolha de um ou mais trabalhos de pesquisa (na forma de artigo, relatório de pesquisa, livro ou trabalho publicado em evento) para representar o tema de busca na base de dados da biblioteca (catálogo online) ou na BVS. Durante a entrevista, os pesquisadores explicaram todo o contexto do tema e os alunos registraram os termos do vocabulário especializado utilizado, pertinente ao tema. Finalmente, os alunos convidaram os pesquisadores a realizar uma busca no catálogo online (Biblioteca) ou na base de dados (BVS) para observar o vocabulário especializado utilizado pelos pesquisadores. Esta sequência de entrevista e observação pelo grupo de alunos tem a finalidade de aumentar o conhecimento prévio sobre o tema de pesquisa para garantir o domínio do assunto durante a indexação de textos sobre o mesmo tema de pesquisa.

2. Dinâmica dentro de sala de aula com pares de alunos - Indexação de artigos ou livros: a classe de 35 alunos se organizou em pares em cada computador para indexar artigos de periódicos científicos (grupos da BVS) e livros (grupos da biblioteca da UNESP), utilizando o material compilado sobre o tema do grupo de pesquisa selecionado e entrevistado para a elaboração do portfólio. Após a indexação, redigiram relato de dificuldades na interação entre pares de alunos. Este exercício foi realizado em sala de aula com interação do professor entre os pares de alunos para exteriorização das dificuldades, uso de procedimentos e estratégias.

\section{ANÁLISE DOS RESULTADOS}


Para efeito de apresentação, os resultados de um grupo de alunos serão tomados como exemplo e demonstrados a partir das duas dinâmicas. $\mathrm{Na}$ dinâmica de Elaboração do Portfólio, apresentaremos o trabalho do Grupo de alunos que realizou o diagnóstico da política de indexação e recuperação da Biblioteca da UNESP, seguida da dinâmica de Indexação realizada por dois alunos do grupo, com base nos resultados da dinâmica do Portfólio. Na demonstração a seguir, apresentamos a parte do trabalho que se refere à sequência de observação e entrevista do usuário da biblioteca pelos alunos com a finalidade específica de aumentar o conhecimento prévio sobre o domínio de Ciências Sociais. O usuário observado e entrevistado no trabalho é um pesquisador de iniciação científica do domínio de Ciências Sociais:

\section{Dinâmica de elaboração do Portfólio}

\section{Pesquisa do usuário entrevistado}

Tema: A Influência do confucionismo no recente processo de desenvolvimento econômico da China. Estudante do $3^{\circ}$ ano de Ciências Sociais da Faculdade de Filosofia e Ciências.

Orientador da UNESP.

Resumo: O Partido Comunista Chinês (PCCh) se vale da tradição de disciplina e de trabalho da doutrina de Confúcio para mobilizar a população para seu projeto político-econômico, mais do que os próprios princípios do marxismo-leninismo ou até mesmo da interpretação destas teorias feitas por Mao Zedong. Qual seria, pois, o papel da doutrina confuciana dentro desse longo projeto chinês, para que, mesmo com o atual nível de crescimento econômico e conseqüentemente um visível aumento na desigualdade da distribuição de renda, se mantenha uma certa "harmonia social", necessária para o sucesso do projeto de enriquecimento chinês. Nossa proposta de estudo, portanto, busca entender a relação dessa doutrina milenar com o presente crescimento econômico chinês, para que possamos contribuir para uma literatura que discuta um tema relevante, visto sua atualidade e dimensão, sendo a China o país que hoje mais cresce economicamente e o confucionismo uma doutrina que há tempos vem influenciando governos de cultura oriental. Para atingirmos os objetivos desta pesquisa, fazemos uso da análise bibliográfica acerca dos principais conceitos confucianos que tratam de questões como harmonia social, a idéia de junzi (cavalheiro) que está totalmente ligada à sociedade estática que Confúcio defende. O Cavalheiro é o homem ideal na visão confucionista, comedido em todas as suas atitudes, respeitoso em relação aos familiares e ancestrais, enfim um homem ideal para que se haja uma sociedade estática. Nosso estudo contempla, como não poderia deixar de ser, as diretrizes político-econômicas atuais da China, para que, dessa maneira, possamos confrontar o resultado da análise conjuntural recente com as idéias confucianas, ilustrando através de uma breve investigação da situação social chinesa.

Palavras-chaves: Mauísmo-marxismo-leninismo; China; Confucionismo; Desenvolvimento econômico.

\section{Estratégia de busca do usuário no catálogo da biblioteca observada pelos alunos:}

- A busca no catálogo, pelo usuário, iniciou-se pela palavra-chave Confúcio;

- O aluno encontrou cinco livros com esse termo e selecionou apenas um;

- Ao utilizar a palavra China, obteve como resultado 74 itens, mas examinou apenas os 20 primeiros e desses selecionou apenas um;

- Com a palavra chave Revolução Chinesa, o usuário obteve 396 itens, dos quais selecionou um item. 


\section{Busca de termos específicos do domínio, pelo usuário, na linguagem de indexação BIBLIODATA}

- Termos principais: Confúcio; Confúcio, Doutrina de; Filosofia chinesa.

Com os livros selecionados na busca ao catálogo, o usuário identificou os termos a serem pesquisados na linguagem de indexação, com a finalidade de aumentar o conhecimento do vocabulário. O primeiro termo procurado foi "Confúcio", depois de observado o título, sumário, orelha do livro, folha de rosto, capa e contracapa. O termo encontrado a partir de "Confúcio" foi "Filosofia chinesa". O terceiro termo encontrado foi "Calvo, Doutrina de". Este termo serve como exemplo de doutrinas, como Confúcio tem uma doutrina, pode-se usar "Confúcio, Doutrina de", é um termo principal.

\section{Indexação realizada pela bibliotecária do livro selecionado pelo usuá rio.}

- Pesquisa por identificação de assunto autorizado no BIBLIODATA pelo termo "Filosofia Chinesa".

- Seleção do termo "Filosofia chinesa".

\section{Dinâmica de indexação}

Textos selecionados, durante a busca pelo usuário, para realizar o exercício de indexação:

\section{Texto 1:}

FERREIRA, Mucio Porphyrio (org.). Confucio : vida e doutrina os analectos. 10 ed. São Paulo:

Pensamento, 2001.

Termos identificados pelos alunos na indexação:

Filosofia chinesa; Confucionismo; Vida; Doutrina; Revolução Chinesa; Confúcio; Filosofia; China; Filosofia de Confúcio

\section{Texto 2:}

YUTANG, Lin. A sabedoria de Confúcio. Rio de Janeiro: José Olympio, 1958.

Termos identificados pelos alunos na indexação:

Confúcio; História de vida; Teorias de Confúcio, Confúcio; Harmonia social; Doutrina de Confúcio; China; Sabedoria; Doutrina confuciana; Enriquecimento Chinês.

\subsection{Análise dos resultados da dinâmica pelo grupo de alunos:}

Pela observação da pesquisa do usuário no catálogo, verifica-se que seu conhecimento de busca assistida e avançada na interface do catálogo é limitado, o que prejudicou o resultado da sua pesquisa. Verifica-se também que utilizou termos genéricos com significados mais abrangentes (China, Revolução Chinesa e Confúcio) para selecionar a busca na base de dados, 
tornando-se prejudicial para sua pesquisa, pois acabou resultando em uma alta revocação ${ }^{1}$ de itens que não foram sequer lidos.

A indexação realizada pela bibliotecária destacou os termos encontrados na ficha catalográfica existente no catálogo: Confúcio, Confucionismo, Filosofia Confucionista e incluiu o termo Filosofia Chinesa.

A linguagem de indexação BIBLIODATA mostrou-se inadequada ao tratar de inclusão de assuntos, pois, muitas vezes, o indexador identifica o assunto como sendo essencial para a pesquisa do usuário, mas o sistema não autoriza seu uso. Contudo, a linguagem de indexação é importante para a padronização dos registros, qualidade, consistência e credibilidade do catálogo.

Portanto, conclui-se, através das entrevistas e indexações realizadas pelas bibliotecárias, que o indexador tem papel fundamental na recuperação da informação desejada pelo usuário, pois mesmo tendo que seguir os assuntos autorizados pelo BIBLIODATA, tem total liberdade de inserir no campo 690 um assunto no qual acreditam que o usuário utilizaria na busca.

O resultado da dinâmica de indexação e a análise do grupo de alunos sobre as duas dinâmicas, revela que a metodologia utilizada para contato de indexadores aprendizes com um contexto profissional real é válida para o ensino de procedimentos de indexação, tendo em vista que o indexador aprendiz interagiu com o contexto analisado, ganhou autonomia na tarefa de indexação, refletiu e soube analisar com objetividade os resultados obtidos.

\section{CONSIDERAÇÕES FINAIS}

Os resultados obtidos desta pesquisa sobre investigação de estratégias de ensino em leitura documentária demonstram que os conhecimentos teóricos e metodológicos, em perspectiva sociocognitiva, proporcionam a visão de um contexto profissional no qual o indexador aprendiz tem consciência de suas estratégias de leitura documentária e consegue desenvolver seu conhecimento prévio profissional, de modo a realizar a tarefa de indexação compatível com a demanda informacional de usuários.

\footnotetext{
1 “A Revocação, ou "recall” ou mesmo "abrangência", é a razão do número de documentos atinentes recuperados sobre o total de documentos atinentes disponíveis na base de dados. A revocação mede o sucesso do SRI em recuperar documentos pertinentes" (SOUZA, 2006, p.163). A alta revocação é proporcionada pela exaustividade na indexação e na recuperação com o uso de termos mais abrangentes. A relação de revocação e precisão é o ideal na recuperação, pois combina o uso de termos mais específicos para recuperar documentos pertinentes em relação à necessidade de informação.
} 
Nesse sentido, a pesquisa desenvolveu o estudo da vinculação do contexto profissional em abordagem sócio-cognitiva, contendo proposta de ensino para articulação do contexto profissional para explorar o contexto de realização das tarefas de indexação em sistemas de tratamento da informação, com ênfase em bibliotecas universitárias.

Dessa forma, recomendamos que a aprendizagem de metodologias de análise de assunto para indexação, pelos alunos, seja antecedida por experiências de convívio com sistemas de recuperação da informação, para contato com demandas reais de usuários, o que exigirá a ampliação da investigação de procedimentos de observação do contexto sócio-cognitivo de leitores profissionais com objetivos de tratamento temático de conteúdos documentários.

THE PROFESSIONAL CONTEXT OF THE INDEXER IN THE TEACHING OF INDEXING

\begin{abstract}
It is proposed the search of teaching methodology with socio-cognitive approach to present teaching strategies that simulate the experience of professional context that is propitious to work out the tasks of subject analysis for the indexing of documents. Taking into account the contact of the learner indexers with the actual professional context, the proposals of Fourie Portfolio (2002) and Hjørland (2002) principle of the information science social context were applied in the discipline "Indexing" at UNESP Librarianship course in Marilia, SP, Brazil. The results taken from this research on teaching strategies in documentary reading show that the methodological and theoretical knowledge, in socio-cognitive perspective, provide a view of a professional context in which the learner indexer is conscious of his strategies of documentary reading and can develop his previous professional knowledge in order to accomplish the indexing tasks compatible to the user informational demand.
\end{abstract}

Keywords: Indexing. Indexing teaching. Socio-cognitive approach. Professional context.

\title{
REFERÊNCIAS
}

ALBRECHTSEN, H. Subject analysis and indexing: from automated indexing to domain analysis. The Indexer, London, v.18, n.4, p. 219-224, oct. 1993.

ARAÚJO, M. de F. A. C. Leitura: um modelo teórico e (algumas) proposta de uma prática consistente. Revista Milenium Online, Viseu, n. 8, oct. 1997 Disponível em: $<$ http://www.ipv.pt/millenium/arq8_1.htm > Acesso em 19/07/2003.

FOURIE, I. How can we take a socio-cognitive approach in teaching indexing and abstracting? The Indexer, London, v. 23, n. 2, p. 83-85, oct. 2003. 
FUJITA, M. S. L. A abordagem cognitiva da leitura como prática pedagógica no ensino da disciplina leitura documentária no curso de Biblioteconomia da UNESP - Campus de Marília: uso do protocolo verbal para metacognição do indexador aprendiz. In: SANTOS, Jussara Pereira Santos (Org.). A leitura com prática pedagógica na formação do profissional da informação. Rio de Janeiro: Fundação Biblioteca Nacional, 2007a. p. 101-132.

La enseñanza de la lectura documentaria en el abordaje cognitivo y socio-cognitivo: orientaciones a la formación del indizador. Anales de Documentación, v.10, p.1 - 16, $2007 \mathrm{~b}$.

FUJITA, M. S. L., RUBI, M. P., BOCCATO, V. R. C. O contexto sociocognitivo do catalogador em bibliotecas universitárias: perspectivas para uma política de tratamento da informação documentária. Datagramazero, v.10, p.1-24, 2009.

GARCIA MARCO, F. J. Los contenidos y la secuencia docente de la Organización del conocimiento. Scire, Zaragoza, v. 1, n. 1, p. 219-228, 1995.

GIASSON, J. A compreensão na leitura. Lisboa: Asa, 1993. 317 p.

GOODMAN, K. S. Miscue analysis: application to reading instruction. Urbana, Ill.: Eric Clearinghouse on Reading and Communication Skills, 1977. Apud ARAÚJO, M. de F. A. C. Leitura: um modelo teórico e (algumas) proposta de uma prática consistente. Revista Milenium Online, Viseu, n.8, oct. 1997 Disponível em: <http://www.ipv.pt/millenium/arq8_1.htm > Acesso em 19/07/2003.

HJØRLAND, B. Information seeking and subject representation: an activity-theoretical approach to information science. Westport: Greenwood Press, 1997. 213p.

HJØRLAND, B. Epistemology and the sócio-cognitive perspective in information science. Journal of the American Society for Information Science and Technology, v. 53, n. 4, p. 257-70, 2002.

HJØRLAND, B., ALBRECHTSEN, H.Toward a new horizon in information science: domainanalysis. Journal of the American Society for Information Science, v.46, n.6, p.400-425, 1995.

SOUZA, R. R. Sistemas de recuperação de informações e mecanismos de busca na web: panorama atual e tendências. Perspectivas em Ciência da Informação, Belo Horizonte, v.11, n.2, p.161-179, maio/ago.2006.

Originais recebidos em: 08/12/2009

Aceito para publicação em: 21/06/2010 